\title{
BIOMARKERS: AN IMPERATIVE ACCESSION FOR DIAGNOSIS OF A DISEASE AND DRUG DEVELOPMENT
}

\author{
MOHAMMAD GULSHAN*, NADENDLA RAMA RAO \\ Department of Pharmaceutics, Chalapathi Institute of Pharmaceutical Sciences, Lam, Guntur, Andhra Pradesh, India. \\ Email: gulshan.md210@gmail.com
}

Received: 07 February 2017, Revised and Accepted: 28 February 2017

\begin{abstract}
Biomarkers are becoming an essential part of clinical development, not least because they offer a faster alternative to the conventional drug development approach and the promise of safer drugs, in greater numbers, approved more quickly. Many of the failures happen late in clinical trials, with the consequence that expenditure in clinical drug development - already a mammoth effort requiring a huge amount of money, time, and patient is increasing. The ultimate vision is to have access to therapeutic fields, a better understanding of pathophysiology of diseases, thereby uncovering potential drug targets and biomarkers in the disease pathway. By finding molecular biomarkers of the disease, diagnosis could be improved and could reveal new information about the disease, by which a better chance for developing drugs is possible. Biomarkers can also reflect the entire spectrum of disease from the earliest manifestations to the terminal stages. In this present review, biomarkers of various diseases were enlisted to highlight the overabundance of information necessary for clinicians and scientists to have a thorough understanding of biomarkers and its ability to improve treatment and reduce health-care costs which are potentially greater than in any other area of medical research.
\end{abstract}

Keywords: Biomarkers, Disease, Pathophysiology, Therapeutics, Treatment.

(C) 2017 The Authors. Published by Innovare Academic Sciences Pvt Ltd. This is an open access article under the CC BY license (http://creativecommons. org/licenses/by/4. 0/) DOI: http://dx.doi.org/10.22159/ajpcr.2017.v10i5.17559

\section{INTRODUCTION}

In 1998, the National Institutes of Health Biomarkers Definitions Working Group defined a biomarker as "a characteristic that is objectively measured and evaluated as an indicator of normal biological processes, pathogenic processes, or pharmacologic responses to a therapeutic intervention" [1,2]. A joint venture on chemical safety, the International Programmed on Chemical Safety, led by the World Health Organization (WHO) and in coordination with the United Nations and the International Labor Organization, has defined a biomarker as "any substance, structure, or process that can be measured in the body or its products and influence or predict the incidence of outcome or disease" [3]. The WHO also stated that a true definition of biomarkers includes "almost any measurement reflecting an interaction between a biological system and a potential hazard, which may be chemical, physical, and biological. The measured response may be functional and physiological, biochemical at the cellular level, or a molecular interaction" [4]. Examples of biomarkers include everything from pulse and blood pressure through basic chemistries to more complex laboratory tests of blood and other tissues $[5,6]$.

Disease-related biomarkers give an indication of whether there is a threat of disease (risk indicator or predictive biomarkers) if a disease already exists (diagnostic biomarkers), or how such a disease may develop in an individual case (prognostic biomarker). Drug-related biomarkers indicate whether a drug will be effective in a specific patient and how the patient's body will process it.

Biomarkers a measure of a normal biological process in the body, a pathological process, or the response of the body to therapy - may offer information about the mechanism of action of the drug, its efficacy, its safety, and metabolite profile. Because biomarkers can predict drug efficacy more quickly than conventional clinical end-points, they hold the potential to substantially accelerate product development in certain disease areas. And because they help to identify earlier those candidates that are likely to fail, they reduce drug development costs, giving life to the concept of "fail early, fail cheap."
Biomarkers have impacted on internal decision-making, i.e., whether to move forward to the next phase of clinical development or not. The decision to move to next phase depends not only on biomarker evidence alone but also they can offer strong supporting evidence, and in the future, it will be the key data in certain programs and offers an objective, biological indicator, rather than just seeing whether the patients feel better. In the present scenario, there is no possibility of developing a new drug without simultaneously looking for biomarkers for efficacy, safety, and to measure the pharmacodynamics of the drug. Mechanistic or target biomarkers can be used in the pre-clinical or phase I trials to measure the pharmacological effect of the drug, i.e., whether the drug interacts with its receptor, enzyme, or protein target, whether it is distributed to the site where it needs to act, whether there is some

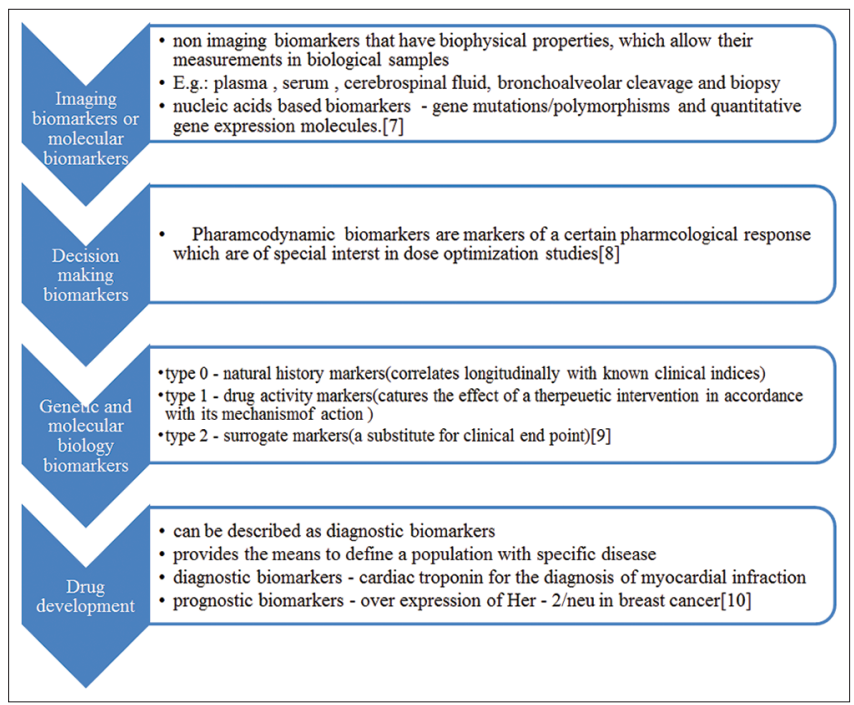

Fig. 1: Classification of biomarkers 
form of downstream pharmacology, and the dose ranges, in which the drug is pharmacologically active. Hence, these types of biomarkers can be used to drive critical go/no - go decisions in drug development (Tables 1 and 2, Figs. 1-4).
At the onset of cancer, a selective protein or gene-based biomarker gets elevated or modified in body fluids or tissues. Early diagnosis of these markers can greatly improve the survival rate or facilitate effective treatment with different modalities. Although the

Table 1: Techniques available for biomarker development

\begin{tabular}{|c|c|c|c|}
\hline Technology & Method & Objective & Tissue \\
\hline Genomics & $\begin{array}{l}\text { SNP genotyping, positional cloning/microsatellites, } \\
\text { expression analyses }\end{array}$ & $\begin{array}{l}\text { Identify susceptibility or disease-modifying } \\
\text { gene } \\
\text { Fine mapping/sequencing of disease loci } \\
\text { Identification of different expression of genes } \\
\text { and signaling pathways }\end{array}$ & $\begin{array}{l}\text { Nucleated cells, } \\
\text { diseased tissue }\end{array}$ \\
\hline Proteomics & 2DGE, MS, LC-MS, GC-MS, MS-MS, MALDI-TOF MS & $\begin{array}{l}\text { Identification of low - abundance proteins, } \\
\text { their subcellular location, post-translational } \\
\text { modification, interactions among proteins }\end{array}$ & $\begin{array}{l}\text { Urine, blood, } \\
\text { saliva, tissues }\end{array}$ \\
\hline Metabolomics & NMR spectroscopy, MS, infrared spectroscopy & $\begin{array}{l}\text { Small molecule identification and } \\
\text { characterization }\end{array}$ & As above \\
\hline Pharmacogenetics & SNP genotyping & Relate genetic makeup to drug response & Nucleated cells \\
\hline Integratomics & All of the above & $\begin{array}{l}\text { Use of high throughput technology to produce } \\
\text { an integrated picture at the DNA, RNA, } \\
\text { protein, tissue, and pharmacological levels }\end{array}$ & All of the above \\
\hline Bioinformatics & BLAST, hierarchical clustering, SOM & Link microarray data to biological pathways & $\begin{array}{l}\text { Data from various } \\
\text { techniques }\end{array}$ \\
\hline
\end{tabular}

MRI: Magnetic resonance imaging, CT: Computed tomography, PET: Positron emission tomography, SPECT: Single-photon emission computed tomography, SNP: Single nucleotide polymorphism, MS: Mass spectroscopy, NMR: Nuclear magnetic resonance, 2DGE: 2D gel electrophoresis, TOF: Time-of-flight, LC-MS: Liquid chromatograph-mass spectroscopy, GC-MS: Gas chromatography-mass spectrometry, SOM: Self-organizing maps

Table 2: Five phases of biomarker development

\begin{tabular}{|c|c|c|c|c|c|}
\hline Phases [14-16] & $\begin{array}{l}\text { Phase } 1 \\
\text { Preclinical } \\
\text { exploratory }\end{array}$ & $\begin{array}{l}\text { Phase } 2 \\
\text { Clinical } \\
\text { characterization and } \\
\text { assay validation }\end{array}$ & $\begin{array}{l}\text { Phase } 3 \\
\text { Clinical association: } \\
\text { Retrospective } \\
\text { screening studies }\end{array}$ & $\begin{array}{l}\text { Phase } 4 \\
\text { Clinical association: } \\
\text { Retrospective } \\
\text { screening studies }\end{array}$ & $\begin{array}{l}\text { Phase } 5 \text { disease } \\
\text { control }\end{array}$ \\
\hline Objective & $\begin{array}{l}\text { Target biomarker } \\
\text { identification, feasibility }\end{array}$ & $\begin{array}{l}\text { Study assay in people } \\
\text { with and without disease }\end{array}$ & $\begin{array}{l}\text { Case-control studies } \\
\text { using respiratory } \\
\text { specimens }\end{array}$ & $\begin{array}{l}\text { Longitudinal studies } \\
\text { to predict disease }\end{array}$ & Clinical use \\
\hline Site & $\begin{array}{l}\text { Biomarker development } \\
\text { laboratory }\end{array}$ & $\begin{array}{l}\text { Biomarker validation } \\
\text { laboratory }\end{array}$ & $\begin{array}{l}\text { Clinical, epidemiologic } \\
\text { centers }\end{array}$ & Cohort studies & Community \\
\hline Design & Cross-sectional & Cross-sectional & Case-control & Prospective & RCT \\
\hline Sample size & Small & Small & Modest & Medium & Large \\
\hline Validity & $\begin{array}{l}\text { Content and construct } \\
\text { validity }\end{array}$ & Criterion validity & Predictive validity & Efficacy of strategy & Effectiveness \\
\hline Result & $\begin{array}{l}\text { Assay, precision, } \\
\text { reliability, sensitivity }\end{array}$ & $\begin{array}{l}\text { Reference limits, } \\
\text { intra-individual variation }\end{array}$ & $\begin{array}{l}\text { Screening characteristics, } \\
\text { true and false+rates }\end{array}$ & ROC analyses & $\begin{array}{l}\text { No needed to } \\
\text { screen treat }\end{array}$ \\
\hline
\end{tabular}

RCT: Randomized controlled trial, ROC: Receiver operating characteristic

Table 3: Breaking the barriers to biomarker discovery

\begin{tabular}{l} 
Barrier to cancer biomarker progress $[\mathbf{1 7 , 1 8}]$ \\
\hline Failure to mechanistically tie a blood biomarker to the tumor itself
\end{tabular}

Improper sample handling and tracking; inadequate tissue fixation and body fluid sample preservation that generates bias, false positives, and false negatives

Lack of independent blinded clinical validation with proper controls for specificity and noncancer diseases

Low analytical sensitivity of mass spectrometry-based detection systems that prevent the detection/identification and measurement of low abundance $(<\mathrm{ng} / \mathrm{nl})$ biomarkers emanating from early stage cancer

\section{Emerging successful strategies to break the barrier}

Discovery of the biomarker across a series of experimental animal tumor models

Mechanistically showing a role in tumor genesis or a change after therapy

Validation of the same marker using human samples

Preservation technologies for tissue and body fluid sample collection Uniform protocols for the collection of tissues and body fluids

Molecular measures to verify the reservation of a biological sample Inclusion of independent epidemiologically credentialed and matched cohorts with inflammatory disease, infectious disease, and benign tumors

Nanotechnology-based methods for biomarker capture, preservation, and exclusion of unwanted high-abundance proteins such as albumin can amplify mass spectrometry sensitivity 1000 
sophisticated imaging technologies such as magnetic resonance imaging, positron emission tomography, and computed tomography have the impact of nanotechnology on their improved performance, they are however unsuitable for the early detection of cancer biomarkers or their quantification. Other approaches for cancer diagnosis based on cell morphology and microscopy (biopsies) are too not conclusive for early diagnosis of cancer. The only hope for early diagnosis of cancer in the near future is by the detection of cancer biomarkers using immunoassays/sensors that are reformed by Nanotechnology. Attractive properties of nanoparticles have miraculously lifted up the design, fabrication, and sensitivity and multiplexing of these immunoassays/sensors in biomarker detection (Tables 3-7).

\section{CONCLUSION}

Biomarkers are biological molecules wit physiological characteristics that are more closely linked to the underlying causes of health or disease. Doctors customarily answer the questions

- Is a patient really sick?

- What medicine is necessary?

- In what dosage?

- Is the patient responding to it?

Based on a variety of symptoms which are giving subjective description and uncertain relationship to the disease state are misleading. Biomarkers give doctors a more objective and quantifiable basis for clinical decision-making.

Table 4: Biomarker of cancer disease and their characteristics with examples

\begin{tabular}{|c|c|c|c|}
\hline Cancer & Markers & Characteristics & $\begin{array}{l}\text { Typical } \\
\text { sample }\end{array}$ \\
\hline \multirow[t]{2}{*}{ Prostate } & PSA, total and free & $\begin{array}{l}\text { High sensitivity in all stages; also elevated from some } \\
\text { non-cancer causes }\end{array}$ & Blood [19] \\
\hline & PSMA & Levels tend to increase with age & Blood \\
\hline \multirow[t]{3}{*}{ Breast } & CA 15-3, 27, 29 & $\begin{array}{l}\text { Elevated in benign breast conditions. Either CA } 15-3 \text { or CA } 27 \text {, } \\
29 \text { could be used as marker }\end{array}$ & Blood $[20,21]$ \\
\hline & Estrogen receptors & Overexpressed in hormone-dependent cancer & Tissue [22] \\
\hline & Her-2/neu & $\begin{array}{l}\text { Only } 20 \sim 30 \% \text { of patients are positive to Her- } 2 \text { oncogene that } \\
\text { is present in multiple copies }\end{array}$ & Tissue [23] \\
\hline Lung (non-small cell) & CEA & $\begin{array}{l}\text { Used in combination with NSA to increase specificity, used also } \\
\text { for colon cancer detection }\end{array}$ & Blood [24] \\
\hline Lung (small cell) & NSE & Better sensitivity toward specific types of lung Caner & Blood [25] \\
\hline Bladder & NMP-22, BTA & $\begin{array}{l}\text { NMP-22 assays tend to have greater sensitivity than BTA } \\
\text { assays }\end{array}$ & Urine [26] \\
\hline Pancreatic & CA $19-9$ & $\begin{array}{l}\text { Elevated also in inflammatory bowel disease, sometimes used } \\
\text { as colorectal cancer biomarker }\end{array}$ & Blood [28] \\
\hline $\begin{array}{l}\text { Epithelial ovarian cancer ( } 90 \% \text { of all } \\
\text { ovarian cancer) }\end{array}$ & CA 125 & $\begin{array}{l}\text { High sensitivity in advanced stage; also elevated with } \\
\text { endometriosis, some other diseases and benign conditions }\end{array}$ & Blood [29] \\
\hline \multirow[t]{2}{*}{ Germ cell cancer of ovaries } & CA 72-4 & $\begin{array}{l}\text { No evidence that this biomarker is better than CA- } 125 \text { but may } \\
\text { be useful when used in combination }\end{array}$ & Blood [30] \\
\hline & AFP & Also elevated during pregnancy and liver cancer & Blood [31] \\
\hline \multirow[t]{2}{*}{ Multiple myeloma and lymphomas } & B2M & $\begin{array}{l}\text { Present in many other conditions, including prostate cancer } \\
\text { and renal cell carcinoma }\end{array}$ & Blood [32] \\
\hline & $\begin{array}{l}\text { Monoclonal } \\
\text { immunoglobulins }\end{array}$ & $\begin{array}{l}\text { Overproduction of an immunoglobulin or antibody, usually } \\
\text { detected by protein electrophoresis }\end{array}$ & $\begin{array}{l}\text { Blood, } \\
\text { Urine [33] }\end{array}$ \\
\hline \multirow[t]{2}{*}{ Metastatic melanoma } & S100B & Subunit of the S100 protein family & Serum [34] \\
\hline & TA-90 & $\begin{array}{l}\text { Could be used to monitor patients with high risks of } \\
\text { developing the disease }\end{array}$ & Serum [35] \\
\hline Thyroid & Thyroglobulin & Principal iodoprotein of the thyroid gland & $\begin{array}{l}\text { Serum, } \\
\text { Tissue [36] }\end{array}$ \\
\hline Testicular & hCG & May regulate vascular neoformation through VEGF & Serum [38] \\
\hline WM & $\begin{array}{l}\text { Monoclonal } \\
\text { immunoglobulin M }\end{array}$ & $\begin{array}{l}\text { The larger size and increased concentration of the monoclonal } \\
\text { protein leads to serum hyperviscosity, the most distinguishing } \\
\text { feature of WM }\end{array}$ & $\begin{array}{l}\text { Blood, } \\
\text { Urine [39] }\end{array}$ \\
\hline Lymphomas & B2M & $\begin{array}{l}\text { Present in many other conditions, including prostate cancer } \\
\text { and renal cell carcinoma }\end{array}$ & Serum [40] \\
\hline $\begin{array}{l}\text { Lung (non small cell), epithelial, } \\
\text { colorectal, head and neck, } \\
\text { pancreatic, or breast }\end{array}$ & EGFR (Her-1) & $\begin{array}{l}\text { Binding of the protein to a ligand induces receptor } \\
\text { dimerization and tyrosine autophosphorylation and leads to } \\
\text { cell proliferation }\end{array}$ & Tissue $[41]$ \\
\hline $\begin{array}{l}\text { Colorectal, lung, breast, pancreatic, } \\
\text { and bladder }\end{array}$ & CEA & $\begin{array}{l}\text { Subtle posttranslational modifications might create differences } \\
\text { between tumor CEA and normal CEA }\end{array}$ & Serum [42] \\
\hline T-ALL & PTK7 & $\begin{array}{l}\text { Membrane-bound surface protein of whole cells, and can be } \\
\text { used to detect circulating tumor cells as targets }\end{array}$ & Blood [43] \\
\hline
\end{tabular}

PSA: Prostate-specific antigen, PSMA: Prostate-specific membrane antigen, CA 15-3, 27, 29: Cancer antigen 15-3, 27, 29, CEA: Carcinoembryonic antigen,

NSE: Neuron-specific enolase, NMP: Matritech's nuclear matrix protein, BTA: Bladder tumor antigen, CA 19-9: Carbohydrate antigen 19-9, CA 125: Cancer antigen 125,

CA 72-4: Cancer antigen 72-4, AFP: Alpha-fetoprotein, B2M: Beta-2 microglobulin, TA-90: Tumor-associated glycoprotein antigen, hCG: Human chorionic gonadotropin,

VEGF: Vascular endothelial growth factor, WM: Waldenstrom's macroglobulinemia, T-ALL: T-cell acute lymphoblastic leukemia, EGFR: Epidermal growth factor receptor 
Table 5: Cardiovascular diseases

\begin{tabular}{|c|c|c|c|}
\hline Name of disease & Effects & $\begin{array}{l}\text { Risk } \\
\text { score (\%) }\end{array}$ & Biomarker \\
\hline Homozygous familial hypercholesterolemia & $\begin{array}{l}\text { Premature cardiovascular morbidity and } \\
\text { mortality }\end{array}$ & $10-20$ & TC, LDL cholesterol [44] \\
\hline Hypertriglyceridemia/hypertriglyceridemia & Elevated levels of Lp(a) & 20 & Lipid profile [45] \\
\hline Chronic kidney disease & Elevated levels of $\mathrm{Lp}(\mathrm{a})$ & 10 & Lipid profile [46] \\
\hline Cholelithiasis & $\begin{array}{l}\text { Gallstone formation due to cholesterol } \\
\text { and salts }\end{array}$ & 20 & $\begin{array}{l}\text { LDL cholesterol and small dense } \\
\text { LDL particles [47] }\end{array}$ \\
\hline Atherosclerosis & Arterial obstruction, chest pain & $\begin{array}{l}20-25 \text { ABCA1 } \\
\text { Efflux }\end{array}$ & $\begin{array}{l}\text { PUFA and carbohydrates, serum } \\
\gamma \text {-glutamyl transferase activity, } \\
\text { blood genomic profiling, and } \alpha 4 \beta 7 \\
\text { integrin [49] }\end{array}$ \\
\hline Coronary heart disease & $\begin{array}{l}\text { Monocytosis, high diabetics, } \\
\text { hypertension, and chronic kidney } \\
\text { diseases }\end{array}$ & 20 & $\begin{array}{l}\text { Impaired sterol efflux, efflux capacity } \\
\text { of HDL, myeloperoxidase increasing } \\
\text { circulating HDL [50] }\end{array}$ \\
\hline Dyslipidemia & $\begin{array}{l}\text { Hypoperfusion, high inflammation, and } \\
\text { low BP }\end{array}$ & 10 & $\begin{array}{l}\text { TC, TG, HDL, LDL, and } \\
\text { anthropometric and biochemical } \\
\text { parameters [52] }\end{array}$ \\
\hline Atherosclerotic peripheral arterial disease & Prevalent, morbid, and mortal diseases & $\begin{array}{l}20 \text { shortening } \\
\text { of lumen }\end{array}$ & LDL cholesterol [53] \\
\hline IHD & $\begin{array}{l}\text { Endothelial dysfunction, vascular } \\
\text { inflammation }\end{array}$ & $10-20$ & $\begin{array}{l}\text { Lipids, cholesterol, calcium, and } \\
\text { cellular debris [54] }\end{array}$ \\
\hline $\begin{array}{l}\text { Diastolic dysfunction and diastolic heart } \\
\text { failure }\end{array}$ & Asymptomatic hypertension & 20 & Myocardial remodeling [55] \\
\hline Chronic heart failures & $\begin{array}{l}\text { ADP-induced platelet aggregation, } \\
\text { triglycerides, end-diastolic volume, } \\
\text { end-diastolic dimension, and ventricular } \\
\text { sepal thickness death }\end{array}$ & $15-20$ & $\begin{array}{l}\text { Lipidemic, hemostasiological, and } \\
\text { hemodynamic indicators, Willebrand } \\
\text { factor, and D-dimer [56] }\end{array}$ \\
\hline Myocardial infarction & Very high morbidity, severe pain & $20-25$ & $\begin{array}{l}\text { Circulating microRNAs level in } \\
\text { patients [57] }\end{array}$ \\
\hline Neuronal dysfunction & $\begin{array}{l}\text { Neuronal cell death and } \\
\text { neuroinflammatory }\end{array}$ & $10-15$ & $\begin{array}{l}\text { 27-hydroxycholesterol, plasma HDL, } \\
\text { NAEs [59] }\end{array}$ \\
\hline Transient global cerebral ischemia & $\begin{array}{l}\text { Cardiac arrest and cardiovascular } \\
\text { Problems }\end{array}$ & $5-10$ & $\omega-3$ PUFAs $[60]$ \\
\hline Hypoglycemia & Cardiac implications & $5-10$ & $\begin{array}{l}\text { Elevated levels of } \operatorname{Lp}(a) \text { and low HDL } \\
\text { cholesterol [61] }\end{array}$ \\
\hline $\begin{array}{l}\text { Hypertriglyceridemia/CAD/acute coronary } \\
\text { syndrome }\end{array}$ & $\begin{array}{l}\text { Severe effect on BMR and peripheral and } \\
\text { cardiac circulation }\end{array}$ & $5-10$ & Altered serum lipid [62] \\
\hline HDL metabolism disorders & Severe inflammation and pain & $5-10$ & LDs [63] \\
\hline Nephrotic syndrome & Renal filtration chocked & $5-10$ & $\begin{array}{l}\text { LDL cholesterol, triglycerides, and } \\
\text { Lp(a) [64] }\end{array}$ \\
\hline Fatal myocardial infarction and brain stroke & $\begin{array}{l}\text { Cardiovascular risks, morbidity, and } \\
\text { mortality in elderly men }\end{array}$ & $20-25$ & $\begin{array}{l}\text { Fat-specific protein Fsp27, } \\
\text { FIT proteins, seipin, and } \\
\text { ADP-ribosylation factor } 1 \text {-coat } \\
\text { protein complex I [65] }\end{array}$ \\
\hline Systemic lupus erythematosus & Problem of PCV and hemoglobin & 5 & $\begin{array}{l}\text { Factors, proteins, ions, and } \\
\text { stimulators of heart muscles [66] }\end{array}$ \\
\hline Acute myocardial infarction & $\begin{array}{l}\text { Death of part of myocardial muscles, } \\
\text { central chest pain, and severe crushing }\end{array}$ & $20-25$ & $\begin{array}{l}\text { Serum soluble ST2 and } \\
\text { interleukin-33 [67] }\end{array}$ \\
\hline \multirow{2}{*}{$\begin{array}{l}\text { Hypertension and dyslipidemia, } \\
\text { hypercholesterolemia }\end{array}$} & Cardiovascular risk factors & $15-20$ & TC and LDL [68] \\
\hline & & $5-10$ & BP and LDL-C, high BMI [69] \\
\hline SCVRs & Tachyarrhythmias, bradyarrhythmias & 5 & $\begin{array}{l}\text { LDL-C, HDL-C, TG, ApoAI, and ApoB } \\
\text { Lp(a) [70] }\end{array}$ \\
\hline $\begin{array}{l}\text { AVDs, type } 2 \text { diabetes, or metabolic } \\
\text { syndrome }\end{array}$ & $\begin{array}{l}\text { Increased levels of triglycerides, low } \\
\text { levels of high-density lipoprotein } \\
\text { cholesterol, and postprandial lipemia }\end{array}$ & $20-25$ & MetS [71] \\
\hline Procardiovascular risks, cardiovascular risks & Inflammation, obesity, and thrombosis & $5-10$ & $\begin{array}{l}\text { Sedentary behavior, } \beta \text {-trace protein } \\
\text { from GFR marker [71] }\end{array}$ \\
\hline
\end{tabular}


Table 5: (Continued)

\begin{tabular}{|c|c|c|c|}
\hline Name of disease & Effects & $\begin{array}{l}\text { Risk } \\
\text { score (\%) }\end{array}$ & Biomarker \\
\hline Metabolic lipid disorders & $\begin{array}{l}\text { Circulatory dysfunctions, high BP, } \\
\text { peripheral pain, and high or low BMR }\end{array}$ & $5-10$ & $\begin{array}{l}\text { MALDI-MS, imaging, and lipidomics } \\
\text { for clinical diagnosis, and proteome } \\
\text { analysis [71] }\end{array}$ \\
\hline Ischemic heart disease & Circulatory dysfunctions & $\begin{array}{l}\text { Smoking, } \\
\text { hypertension, } \\
\text { age, family } \\
\text { history }\end{array}$ & $\begin{array}{l}\text { Endothelial dysfunction, monocyte } \\
\text { accumulation, endothelial apoptosis, } \\
\text { and thrombus formation [71] }\end{array}$ \\
\hline Low HDL-C syndromes & Increased risk of CAD & 5 & $\begin{array}{l}\text { Sphingomyelin phosphodiesterase } 1 \\
\text { and glucocerebrosidase [71] }\end{array}$ \\
\hline Hypothyroidism and gall stone & Severe pain, inflammation & 5 & $\begin{array}{l}\text { TSH level and sodium and potassium } \\
\text { salts [71] }\end{array}$ \\
\hline $\begin{array}{l}\text { Multiple CVDs, diabetes, stroke, and } \\
\text { recurrent ischemia syndrome }\end{array}$ & $\begin{array}{l}\text { Hepatic inflammation due to common } \\
\text { carotid intima-media thickness }\end{array}$ & $10-20$ & $\begin{array}{l}\text { Multiple biomarkers, vascular } \\
\text { imaging [71] }\end{array}$ \\
\hline Angina pectoris & $\begin{array}{l}\text { Obesity, arterial thickness, BMI, and } \\
\text { respiration rate, and severe chest pain }\end{array}$ & $10-0$ & Coronary angiography [71] \\
\hline Myocardial infarction & PAPP-A in serum & 15 & $\begin{array}{l}\text { Severe blood pressure changes, } \\
\text { central chest pain, and silent or } \\
\text { knocking angina [71] }\end{array}$ \\
\hline
\end{tabular}

HDL: High-density lipoprotein, TC: Total cholesterol, LDL: Low-density lipoprotein, CVD: Cardiovascular disease, PUFA: Polyunsaturated fat, IHD: Ischemic heart disease, TG: Triglyceride, NAEs: N-acylethanolamines, LDs: Lipid droplets, CAD: Coronary artery disease, FIT: Fat storage-inducing transmembrane, BMI: Body mass index, PAPP-A: Pregnancy-associated plasma protein-A, TSH: Thyroid-stimulating hormone, BMR: Basal metabolic rate, BP: Blood pressure, GFR: Glomerular filtration rate, AVD: Atherosclerotic vascular disease, SCVRs: Spinal cord vascular resistances, MS: Mass spectroscopy, MetS: Metabolic syndrome

Table 6: Hepatocellular carcinoma biomarkers

\begin{tabular}{ll}
\hline HCC marker & Clinical use \\
\hline AFP & Early diagnosis, monitoring, and recurrence \\
Lens culinaris agglutinin reactive AFP (AFP-L3\%) & Early diagnosis and prognosis, vascular invasion \\
DCP & Early diagnosis and prognosis, portal vein invasion and metastasis \\
Gamma-glutamyl transferase & Early diagnosis complementary to other markers \\
Alpha-l-fucosidase & Early diagnosis \\
Glypican-3 & Early diagnosis \\
Human carbonyl reductase 2 & Prognosis \\
Golgi phosphoprotein 2 & Tumor aggressiveness \\
Transforming growth factor beta & Tumor invasiveness \\
HGF & Prognosis and disease recurrence \\
TGF-b & Prognosis invasiveness \\
Tumor-specific growth factor & Diagnosis complementary to other markers \\
Epidermal growth factor receptor family & Early recurrence \\
Hepatocyte growth factor & Metastasis reduced survival \\
Micro RNAs & Tumor spread and survival [72] \\
\hline
\end{tabular}

AFP: Alpha-fetoprotein, DCP: Des-gamma-carboxy prothrombin, HGF: Hepatocyte growth factor, TGF-b: Transforming growth factor-b, HCC: Hepatocellular carcinoma

Table 7: Analytical method to discover biomarker for Alzheimer's disease diagnosis

\begin{tabular}{ll}
\hline Analytical method & Biomarker \\
\hline ELISA & A $\beta 42$, total tau, \\
phospho - tau - 181 (single) & 16 signaling proteins \\
Multiplex searchlight ELISAs & 18 signaling proteins \\
Filter-based array sandwich ELISA & A 442 , total tau, \\
INNO - BIA AlzBio3 Luminex - based technology (innogenetics) & phospho - tau - 181 (multiplex) \\
& 2325 tissue specimens \\
Tissue array & 33 genes, multiple phosphorylated tau \\
Quantitative real-time RT-PCR & epitopes \\
& A 4 40, A $\beta 42$ \\
Liquid chromatography/electrosprayy ionosation MS & 1000 polypeptides \\
Capillary electrophoresis/MS & Trace elements and metal ions \\
Ultrasensitive laser ablation inductively coupled plasma/MS & 1500 CSF proteins \\
Multiplex iTRAQ & Several A species: \\
Surface-enhanced laser desorption/ionization or matrix-assisted laser desorption/ionization & A 337, A $\beta 36$, A $\beta 38$, A $\beta 40$ \\
& Several thousand genes [73] \\
DNA/RNA chips, biochips, gene chips &
\end{tabular}

MS: Mass spectroscopy, CSF: Cerebrospinal fluid, RT-PCR: Real-time polymerase chain reaction 


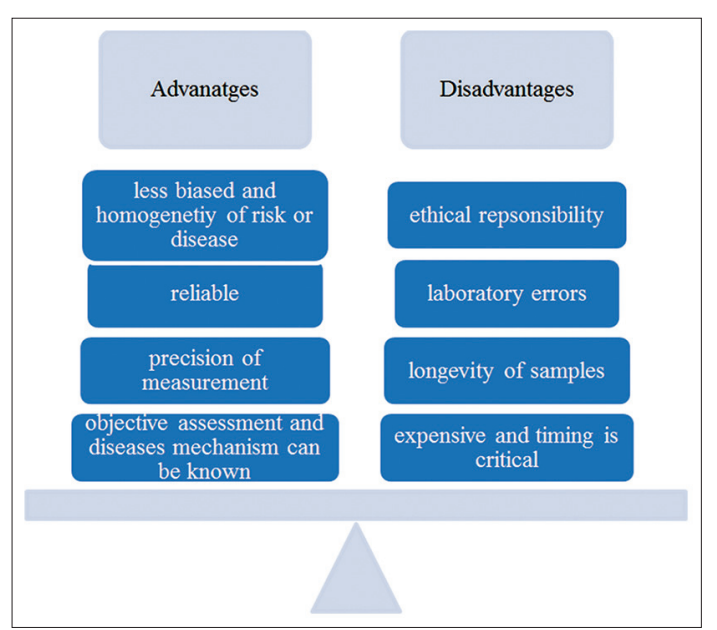

Fig. 2: Relative advantages and disadvantages of biomarkers [11]

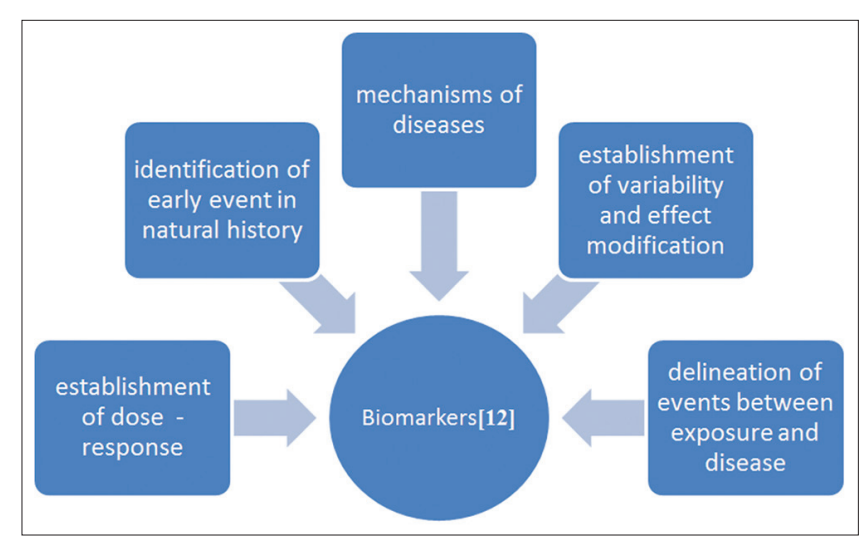

Fig. 3: Salient features of biomarkers

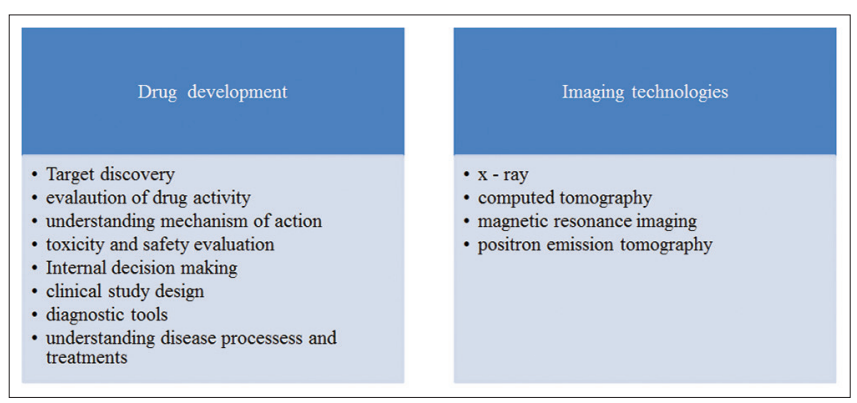

Fig. 4: Biomarkers applications

\section{REFERENCES}

1. Biomarkers Definition Working Group. Biomarkers and surrogate endpoints: Preferred definitions and conceptual framework. Clin Pharmacol Ther 2001;69:89-95.

2. Strimbu K, Tavel JA. What are biomarkers? Curr Opin HIV AIDS 2010;5(6):463-6.

3. WHO International Programme on Chemical Safety. Biomarkers in Risk Assessment: Validity and Validation. 2001. Available from: http:// www.inchem.org/documents/ehc/ehc/ehc222.htm.

4. WHO International Programme on Chemical Safety. Biomarkers and Risk Assessment: Concepts and Principles. 1993. Available from http:// www.inchem.org/documents/ehc/ehc/ehc155.htm.

5. Fleming TR, DeMets DL. Surrogate end points in clinical trials: Are we being misled? Ann Intern Med 1996;125(7):605-13.

6. Desai M, Stockbridge N, Temple R. Blood pressure as an example of a biomarker that functions as a surrogate. AAPS J 2006;8(1):E146-52.

7. Gertrude H. Sergievsky Center and the Taub Institute for Research on Alzheimer's Disease and the Aging Brain. New York: College of
Physicians and Surgeons, Columbia University; 2013.

8. Craig-Schapiro R, Fagan AM, Holtzman DM. Biomarkers of Alzheimer's disease. Neurobiol Dis 2009;35(2):128-40.

9. Mayeux R. New York. Available from: http://www.rpm2/at/columbia. edu.

10. U.S. Food and Drug Administration. Innovation or Stagnation: Challenge and Opportunity on the Critical Path to New Medical Products. Rockville, MD: U.S. Food and Drug Administration, U.S. Department of Health and Human Services; 2004. Available from: http://www.fda. gov/oc/initiatives/criticalpath/whitepaper.html\#execsummary.

11. Mayeux R, Saunders AM, Shea S, Mirra S, Evans D, Roses AD, et al. Utility of the apolipoprotein E genotype in the diagnosis of Alzheimer's disease. Alzheimer's disease centers consortium on apolipoprotein E and alzheimer's disease. N Engl J Med 1998;338(8):506-11.

12. Schulte PA. A conceptual and historical framework for molecular epidemiology. In: Molecular Epidemiology. Principles and Practices. San Diego, CA: Academic Press; 1993. p. 3-44

13. Ilyin SE, Belkowski SM, Plata-Salamán CR. Biomarker discovery and validation: Technologies and integrative approaches. Trends Biotechnol 2004;22(8):411-6.

14. Pepe MS, Etzioni R, Feng Z, Potter JD, Thompson ML, Thornquist M, et al. Phases of biomarker development for early detection of cancer. J Natl Cancer Inst 2001;93(14):1054-61.

15. Barker PE. Cancer biomarker validation: Standards and process: Roles for the national institute of standards and technology (NIST). Ann N Y Acad Sci 2003;983:142-50.

16. Rothman N, Hainaut P, Schulte P, Smith M, Boffetta P, Perera F. Molecular Epidemiology: Principles and Practice. San Diego, California: Academic Press; 1993. p. 79-107.

17. Luchini A, Geho DH, Bishop B, Tran D, Xia C, Dufour RL, et al. Smart hydrogel particles: Biomarker harvesting: One-step affinity purification, size exclusion, and protection against degradation. Nano Lett 2008;8(1):350-61.

18. Taguchi A, Politi K, Pitteri SJ, Lockwood WW, Faça VM, Kelly-Spratt K, et al. Lung cancer signatures in plasma based on proteome profiling of mouse tumor models. Cancer Cell 2011;20(3):289-99.

19. Ludwig JA, Weinstein JN. Biomarkers in cancer staging, prognosis and treatment selection. Nat Rev Cancer 2005;5(11):845-56.

20. Catalona WJ. Clinical utility of measurements of free and total prostatespecific antigen (PSA): A review. Prostate Suppl 1996;7:64-9.

21. Beckett ML, Cazares LH, Vlahou A, Schellhammer PF, Wright GL Jr. Prostate-specific membrane antigen levels in sera from healthy men and patients with benign prostate hyperplasia or prostate cancer. Clin Cancer Res 1999;5(12):4034-40.

22. Zhenhua M, Ma Q, Wang Z. An evaluation of the diagnostic value of CA19-9 and CEA levels in patients with pancreatic cancer. J Nanjing Med Univ 2009;23:199-202.

23. Chen CC, Yang SH, Lin JK, Lin TC, Chen WS, Jiang JK, et al. Is it reasonable to add preoperative serum level of CEA and CA19-9 to staging for colorectal cancer? J Surg Res 2005;124(2):169-74.

24. Ozaksit G, Caglar T, Ciçek N, Kusçu E, Batioglu S, Gökmen O. Serum CA 125 levels before, during and after treatment for endometriosis. Int J Gynaecol Obstet 1995;50(3):269-73.

25. Fayed ST, Ahmad SM, Kassim SK, Khalifa A. The value of CA 125 and CA72-4 in management of patients with epithelial ovarian cancer. Dis Markers 1998;14(3):155-60.

26. Richardson BE, Hulka BS, Peck JL, Hughes CL, van den Berg BJ, Christianson RE, et al. Levels of maternal serum alpha-fetoprotein (AFP) in pregnant women and subsequent breast cancer risk. Am J Epidemiol 1998;148(8):719-27.

27. Bataille R, Grenier J, Commes T. In vitro production of beta 2 microglobulin by human myeloma cells. Cancer Invest 1988;6(3):271-7.

28. Cordoba F, Lavabre-Bertrand T, Salhi SL, Huguet MF, Gerfaux J, Rossi JF, et al. Spontaneous monoclonal immunoglobulin-secreting peripheral blood mononuclear cells as a marker of disease severity in multiple myeloma. Br J Haematol 2000;108(3):549-58.

29. Hauschild A, Engel G, Brenner W, Gläser R, Mönig H, Henze E, et al. Predictive value of serum S100B for monitoring patients with metastatic melanoma during chemotherapy and/or immunotherapy. $\mathrm{Br}$ J Dermatol 1999;140(6):1065-71.

30. Hsueh EC, Gupta RK, Glass EC, Yee R, Qi K, Morton DL. Positron emission tomography plus serum TA90 immune complex assay for detection of occult metastatic melanoma. J Am Coll Surg 1998;187(2):191-7.

31. Pacini F, Pinchera A. Serum and tissue thyroglobulin measurement: Clinical applications in thyroid disease. Biochimie 1999;81(5):463-7.

32. Elisei R. Routine serum calcitonin measurement in the evaluation 
of thyroid nodules. Best Pract Res Clin Endocrinol Metab 2008;22(6):941-53.

33. Arrieta O, Michel Ortega RM, Angeles-Sánchez J, Villarreal-Garza C, Avilés-Salas A, Chanona-Vilchis JG, et al. Serum human chorionic gonadotropin is associated with angiogenesis in germ cell testicular tumors. J Exp Clin Cancer Res 2009;28:120.

34. Vijay A, Gertz MA. Waldenström macroglobulinemia. Blood 2007;109(12):5096-103.

35. Federico M, Guglielmi C, Luminari S, Mammi C, Marcheselli L, Gianelli U, et al. Prognostic relevance of serum beta2 microglobulin in patients with follicular lymphoma treated with anthracycline-containing regimens. A GISL study. Haematologica 2007;92(11):1482-8.

36. Salomon DS, Brandt R, Ciardiello F, Normanno N. Epidermal growth factor-related peptides and their receptors in human malignancies. Crit Rev Oncol Hematol 1995;19(3):183-232.

37. Sidransky D. Emerging molecular markers of cancer. Nat Rev Cancer 2002;2(3):210-9.

38. Hammarström S. The carcinoembryonic antigen (CEA) family: Structures, suggested functions and expression in normal and malignant tissues. Semin Cancer Biol 1999;9(2):67-81.

39. Shangguan D, Li Y, Tang Z, Cao ZC, Chen HW, Mallikaratchy P, et al. Aptamers evolved from live cells as effective molecular probes for cancer study. Proc Natl Acad Sci U S A 2006;103(32):11838-43.

40. Henderson IC, Patek AJ. The relationship between prognostic and predictive factors in the management of breast cancer. Breast Cancer Res Treat 1998;52(1-3):261-88.

41. Dandachi N, Dietze O, Hauser-Kronberger C. Chromogenic in situ hybridization: A novel approach to a practical and sensitive method for the detection of HER2 oncogene in archival human breast carcinoma. Lab Invest 2002;82(8):1007-14.

42. Molina R, Auge JM, Escudero JM, Marrades R, Viñolas N, Carcereny E, et al. Mucins CA 125, CA 19.9, CA 15.3 and TAG-72.3 as tumor markers in patients with lung cancer: Comparison with CYFRA 21-1, CEA, SCC and NSE. Tumour Biol 2008;29(6):371-80.

43. Landman J, Chang Y, Kavaler E, Droller MJ, Liu BC. Sensitivity and specificity of NMP-22, telomerase, and BTA in the detection of human bladder cancer. Urology 1998;52(3):398-402.

44. Kalantarian S, Rimm EB, Herrington DM, Mozaffarian D. Dietary macronutrients, genetic variation, and progression of coronary atherosclerosis among women. Am Heart J 2014;167(4):627-35.e1.

45. Shao B, Tang C, Sinha A, Mayer PS, Davenport GD, Brot N, et al. Humans with atherosclerosis have impaired ABCA1 cholesterol efflux and enhanced high-density lipoprotein oxidation by myeloperoxidase. Circ Res 2014;114(11):1733-42.

46. Distiller LA. Why do some patients with type 1 diabetes live so long? World J Diabetes 2014;5(3):282-7.

47. Mueller T, Hinterreiter F, Luft C, Poelz W, Haltmayer M, Dieplinger B. Mortality rates and mortality predictors in patients with symptomatic peripheral artery disease stratified according to age and diabetes. J Vasc Surg 2014;59(5):1291-9.

48. Unis A, Abdelbary A, Hamza M. Comparison of the effects of escitalopram and atorvastatin on diet-induced atherosclerosis in rats. Can J Physiol Pharmacol 2014;92(3):226-33.

49. Hadi NR, Mohammad BI, Ajeena IM, Sahib HH. Antiatherosclerotic potential of clopidogrel: Antioxidant and anti-inflammatory approaches. Biomed Res Int 2013;2013:790263.

50. Collier P, Watson CJ, Voon V, Phelan D, Jan A, Mak G, et al. Can emerging biomarkers of myocardial remodelling identify asymptomatic hypertensive patients at risk for diastolic dysfunction and diastolic heart failure? Eur J Heart Fail 2011;13:1087-95.

51. Kachkovskii MA, Simerzin VV, Rybanenko OA, Kirichenko NA. Hemostasiological, lipidemic, and hemodynamic indicators associated with the risk of cardiovascular death in high- and very high-risk patients according to the SCORE scale. Ter Arkh 2014;86(3):59-64.

52. Whelton SP, Narla V, Blaha MJ, Nasir K, Blumenthal RS, Jenny NS, et al. Association between resting heart rate and inflammatory biomarkers (High-sensitivity C-reactive protein, interleukin-6, and fibrinogen) (From the multi-ethnic study of atherosclerosis). Am J
Cardiol 2014;113(4):644-9.

53. Rubenfire M, Brook RD. HDL cholesterol and cardiovascular outcomes: What is the evidence? Curr Cardiol Rep 2013;15(4):349.

54. Catapano AL, Pirillo A, Bonacina F, Norata GD. HDL in innate and adaptive immunity. Cardiovasc Res 2014;103(3):372-83.

55. Zampetaki A, Willeit P, Tilling L, Drozdov I, Prokopi M, Renard JM, et al. Prospective study on circulating MicroRNAs and risk of myocardial infarction. J Am Coll Cardiol 2012;60(4):290-9.

56. von Schacky C. Omega-3 index and cardiovascular health. Nutrients 2014;6(2):799-814.

57. Heringlake M, Charitos EI, Gatz N, Käbler JH, Beilharz A, Holz D, et al. Growth differentiation factor 15: A novel risk marker adjunct to the EuroSCORE for risk stratification in cardiac surgery patients. J Am Coll Cardiol 2013;61(6):672-81

58. Grundy SM. Use of emerging lipoprotein risk factors in assessment of cardiovascular risk. JAMA 2012;307(23):2540-2.

59. Luo C, Ren H, Wan JB, Yao X, Zhang X, He C, et al. Enriched endogenous omega-3 fatty acids in mice protect against global ischemia injury. J Lipid Res 2014;55(7):1288-97.

60. Esposito E, Cordaro M, Cuzzocrea S. Roles of fatty acid ethanolamides (FAE) in traumatic and ischemic brain injury. Pharmacol Res 2014;86:26-31

61. Hanefeld M, Duetting E, Bramlage P. Cardiac implications of hypoglycaemia in patients with diabetes - a systematic review. Cardiovasc Diabetol 2013;12:135.

62. Tan JS, Seow CJ, Goh VJ, Silver DL. Recent advances in understanding proteins involved in lipid droplet formation, growth and fusion. J Genet Genomics 2014;41(5):251-9.

63. Gupta S, Gudapati R, Gaurav K, Bhise M. Emerging risk factors for cardiovascular diseases: Indian context. Indian J Endocrinol Metab 2013;17(5):806-14

64. Rich-Edwards JW, Fraser A, Lawlor DA, Catov JM. Pregnancy characteristics and women's future cardiovascular health: An underused opportunity to improve women's health? Epidemiol Rev 2014;36:57-70.

65. Hodcroft CJ, Rossiter MC, Buch AN. Cannabis-associated myocardial infarction in a young man with normal coronary arteries. J Emerg Med 2014;47(3):277-81

66. Weiner SD, Ahmed HN, Jin Z, Cushman M, Herrington DM, Nelson JC, et al. Systemic inflammation and brachial artery endothelial function in the multi-ethnic study of atherosclerosis (MESA). Heart 2014;100(11):862-6.

67. Hurks R, Vink A, Hoefer IE, de Vries JP, Schoneveld AH, Schermerhorn ML, et al. Atherosclerotic risk factors and atherosclerotic postoperative events are associated with low inflammation in abdominal aortic aneurysms. Atherosclerosis 2014;235(2):632-41.

68. Oni ET, Agatston AS, Blaha MJ, Fialkow J, Cury R, Sposito A, et al. A systematic review: Burden and severity of subclinical cardiovascular disease among those with nonalcoholic fatty liver; should we care? Atherosclerosis 2013;230(2):258-67.

69. Nursalim A, Suryaatmadja M, Panggabean M. Potential clinical application of novel cardiac biomarkers for acute myocardial infarction. Acta Med Indones 2013;45(3):240-50.

70. Eggers KM, Al-Shakarchi J, Berglund L, Lindahl B, Siegbahn A, Wallentin $\mathrm{L}$, et al. High-sensitive cardiac troponin $\mathrm{T}$ and its relations to cardiovascular risk factors, morbidity, and mortality in elderly men. Am Heart J 2013;166(3):541-8

71. O’Malley RG, Bonaca MP, Scirica BM, Murphy SA, Jarolim P, Sabatine MS, et al. Prognostic performance of multiple biomarkers in patients with non-ST-segment elevation acute coronary syndrome: Analysis from the MERLIN-TIMI 36 trial (Metabolic efficiency with ranolazine for less ischemia in non-ST-elevation acute coronary syndromes-thrombolysis in myocardial infarction 36). J Am Coll Cardiol 2014;63(16):1644-53.

72. Humpel C. Identifying and validating biomarkers for Alzheimer's disease. Trends Biotechnol 2011;29(1):26-32

73. Liotta LA, Petricoin E. Cancer biomarkers: Closer to delivering on their promise. Cancer Cell 2011;20(30):279-80. 